\title{
Minimizing Prosthetic Intervention to Correct Occlusal Plane Discrepancy in a Complex Fixed- Removable Combination Case
}

ISSN: 2637-7764

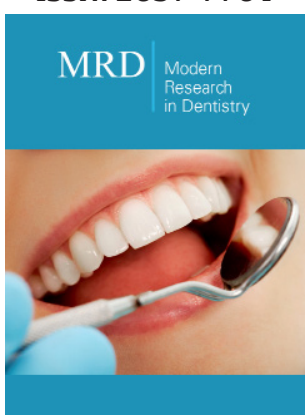

*Corresponding author: EmmanouilGeorgios Tzanakakis, Perikleous 2, Cholargos 15561, Athens, Greece

Submission: 眥 December 22, 2020

Published: 洫January 06, 2021

Volume 6 - Issue 1

How to cite this article: Tzanakakis Emmanouil, Zoidis Panagiotis. Minimizing Prosthetic Intervention to Correct Occlusal Plane Discrepancy in a Complex FixedRemovable Combination Case. Mod Res Dent. 6(1). MRD. 000627. 2021. DOI: 10.31031/MRD.2021.06.000627

Copyright@ Tzanakakis Emmanouil, This article is distributed under the terms of the Creative Commons Attribution 4.0 International License, which permits unrestricted use and redistribution provided that the original author and source are credited.
Tzanakakis Emmanouil1* and Zoidis Panagiotis ${ }^{2}$

${ }^{1}$ Department of Operative Dentistry, National and Kapodistrian University of Athens, Dental School of Athens, Greece

${ }^{2}$ Division of Prosthodontics, Department of Restorative Dental Sciences, University of Florida College of Dentistry, Gainesville USA

\begin{abstract}
Partial edentulism may be the cause of compromised mastication, articulation and changes in esthetic appearance. Although dental implants can provide a viable treatment option to conventional fixed and removable restorations, there are certain reasons that lead patients to reject implant treatment. The conventional prosthodontic approach combining fixed and removable prostheses is well documented and can result in reliable treatment solutions. Prosthetic intervention requires preparation of abutment teeth followed by appropriate prosthetic design in order to re-establish occlusal stability. Such combination full-arch prostheses should preserve health, esthetics and function. The aim of this case report is to present an alternative way to correct occlusal plane discrepancies through a full mouth rehabilitation in a step-by step procedure.
\end{abstract}

Keywords: Attachments;Combined fixed-removable restoration;Metal clasps;Occlusal plane;Removable dental prosthesis

\section{Introduction}

Partial edentulism, if not treated, may lead to complex esthetic and functional problems. Moreover, patients often postpone their prosthetic treatment due to phsycological or financial reasons. Most patients with severe edentulism are unaware of the difficulty and the time that is needed for a proper treatment plan for their prosthetic rehabilitation [1]. Although dental implants can provide additional support both for fixed and removable restorations, and offer viable treatment options, some patients tend to reject implants as a treatment solution. The basic reason would be the widespread misinformation that dental implants require more complicated and time-consuming procedures. Additionally, the higher cost of the surgical procedure and the possible post-operative complications make elderly patients reluctant to implant placement [2].

Conventional prosthodontics involving combination of fixed and removable prostheses are well documented and account for an acceptable and reliable treatment plan. Prosthodontic approach requires preparation of abutment teeth and appropriate prosthetic design in order to re-establish occlusal stability. The combination of fixed and removable prosthodontics is a reasonable treatment plan when extended edentulous areas are present. The design of such full-arch prosthesis should preserve health, esthetics, and function [3].

The removable dental prosthesis (RDP) design is a crucial factor for treatment planning. The selection of major and minor connectors is based on the clinician's experience and knowledge. Visible parts of an RDP should first be acceptable by the patient. The dilemma between clasp retainers and precision attachments is often a matter of controversy. Clasping is well documented, requires less complicated laboratory procedures and is easier when it comes to repairing $[4,5]$. Precision or semi-precision attachments are almost invisible in anterior regions but increase stress to supporting abutment teeth. Moreover, extra coronal 
precision attachments work as cantilevers and increase the risk of abutment failure $[4,6]$. Clinicians often use crown splinting to distribute masticatory forces more favorably between abutment teeth [7-10]. The cost of treatment planning attachments is usually higher than that for conventional clasping and precise laboratory procedures and techniques play a significant role for the longevity of such restorations $[11,12]$.

Occlusal rehabilitation has always been a challenge in such combination cases. Thorough radiographic and intraoral examination as well as study cast analysis are imperative to create a viable treatment plan. Occlusal discrepancies can be evaluated and corrected by means of a full mouth wax up on the diagnostic casts. The final result should reestablish esthetics and function. The aim of this case report is to present the full mouth rehabilitation of a complicated clinical case in a step-by step procedure.

\section{Case History}

A 65-year-old male Caucasian patient presented with a failing dentition. The patient had never before any prosthodontic therapy. He reported previous traumatic experience from dental therapy and claimed that the idea of visiting a dentist caused psychological stress, explaining that he would only visit the dentist as an emergency case. The only dental treatment he had received in the past was the extraction of problematic teeth. Several initial visits were spent to help the patient overcome his negative attitude towards dental treatment.

His medical record was free, except he was a heavy smoker. Dental examination revealed caries, moderate periodontitis, and loss of posterior occlusal support. Periodontal examination revealed gingival inflammation and pocket depths lower than $4 \mathrm{~mm}$. Significant recession areas were identified, and all teeth were characterized with good prognosis. Osseous support averaged 50\% for both maxillary and mandibular teeth (Figure 1).

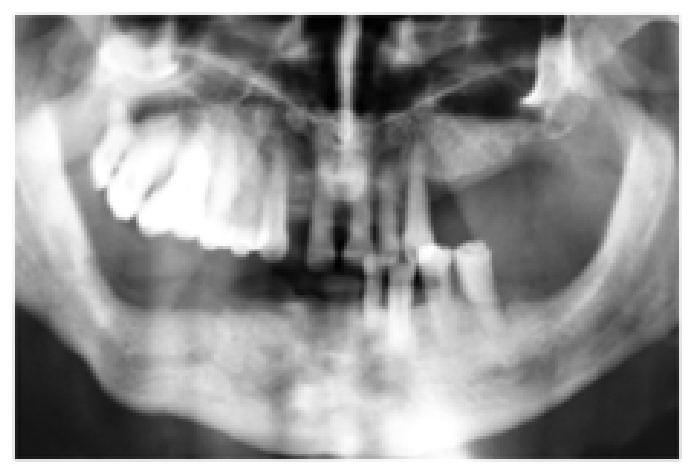

Figure 1: Initial Panoramic radiograph.

The loss of posterior support due to the absence of posterior teeth resulted in occlusal instability. Several teeth were missing in the maxillary left quadrant. The maxillary arch was characterized as Kennedy Class I according to the classification of the American College of Prosthodontics (Figure 2A). A significant long axis distal turn of the maxillary central incisors and severe abrasion of left lateral and canine teeth were also recorded. A significant occlusal plane discrepancy was present, defined by significant overeruption of right second bicuspid and first molar. The mandibular arch was characterized as a modified Kennedy Class I, with only four teeth present (Figure 2B). Caries were present on the left second bicuspid, as well as lingual inclination of left first and second bicuspid and incisal wear on the remaining teeth. The vertical dimension of occlusion (VDO) was slightly decreased (1-2mm) and maximum inter-cuspal position revealed only three occlusal contacts, resulting to occlusal trauma (Figure 2C).

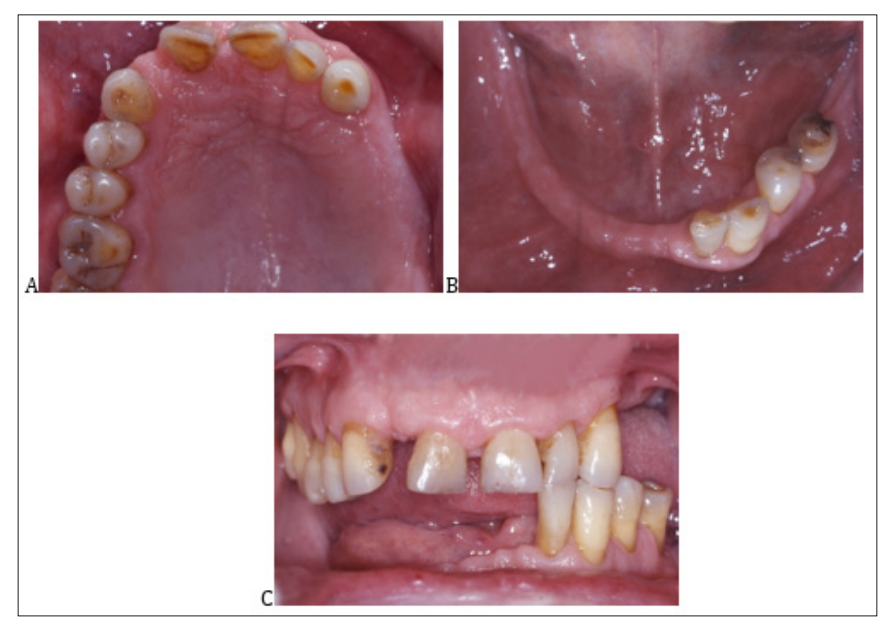

Figure 2: Preoperative condition.
A. Maxilla.
B. Mandible.
C. Frontal view.

The patient was seeking a treatment plan to improve his compromised chewing and speaking ability. Patient rejected the implant option due to increased cost, and his inability to quit smoking. The patient also wanted to avoid a clasp on the canine that would compromise esthetics.

After clinical examination and diagnosis and taking under consideration the above-mentioned restrictions, two different treatment plans were proposed for the maxillary and mandibular arch, combining fixed and removable restorations. In the maxilla, the point of controversy was the occlusal plane correction. The first treatment plan would include occlusal plane correction by significantly reducing right maxillary abutment teeth in order to restore with PFM crowns. This significant tooth reduction would possibly require endodontic therapies. Maxillary anterior teeth would be restored with a six-unit bridge. The fixed restoration would combine with a unilateral removable partial denture.

A second and more conservative treatment plan was implemented in order to correct this inverted occlusal plane. Instead of reducing posteriorly, anterior teeth elongation (2-3mm) was decided. Those teeth would be prepared regardless, in order to fabricate the anterior bridge restoration that would combine 
with the RDP. Concerning this RDP, an extra-coronal semi-precision attachment (ERA; Sterngold Co) was proposed distally to the left canine to improve esthetics [13-15].

In the mandible, the left lateral incisor was planned for extraction due to poor prognosis. The treatment plan included three splinted metal ceramic crowns for the remaining mandibular teeth (left canine and bicuspids) and a RPD. A simple conventional bilateral clasp design was proposed for the RPD since there were no esthetic demands [16-18].

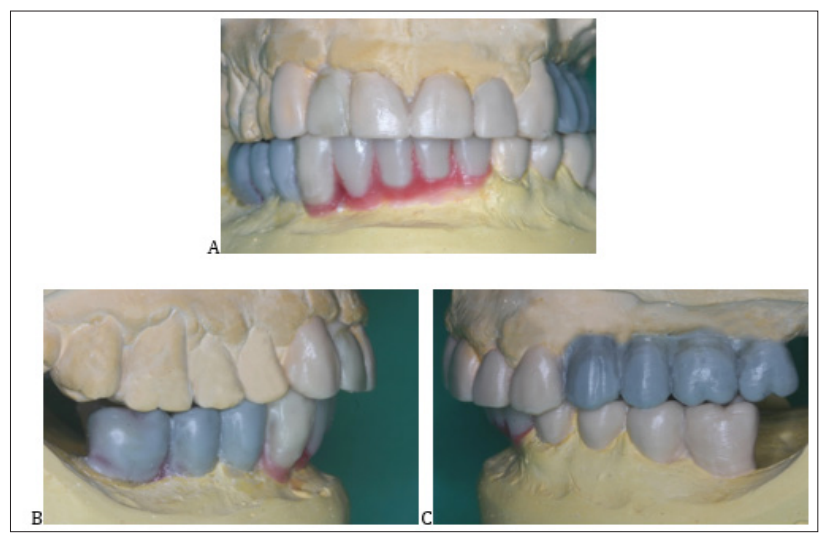

Figure 3: Diagnostic wax-up to verify treatment plan.

A. Frontal view.

B. B, C Lateral views.

A detailed wax-up was performed to justify that this second, conservative approach would be a viable solution (Figures 3A,B,C). Conservative periodontal treatment, followed by the preparation of all maxillary and mandibular abutment teeth was performed. Provisional restorations were fabricated chairside according to the wax up and an interim RDP was delivered to ensure proper posterior support. Provisional restorations were trial tested for three months to ensure esthetic and functional requirements as well as patient's compliance with oral hygiene instructions.

Final impressions were performed using the double mix technique. Occlusal records included face-bow registration and precise centric relation registration. Metal frameworks try-in, verified complete marginal covering and passive fit. Bisque try in, allowed for esthetic adjustments. Definitive RDP impressions were performed using custom trays and polyether impression material. RPDs were also examined for accurate fit. A final try-in stage following RDP processing, allowed for a final occlusal adjustment of both fixed and removable restorations.

The final cementation of the maxillary fixed restorations was performed using conventional Glass-Ionomer cement (Riva, SDI Co, USA). In the mandible, an adhesive protocol was selected and a resin cement (Panavia F2.0; Kuraray Co) was used, due to limited number of abutments and increased expected torque values. The final result fulfilled patient's expectations and demands, significantly improving his smile (Figures 4-6).

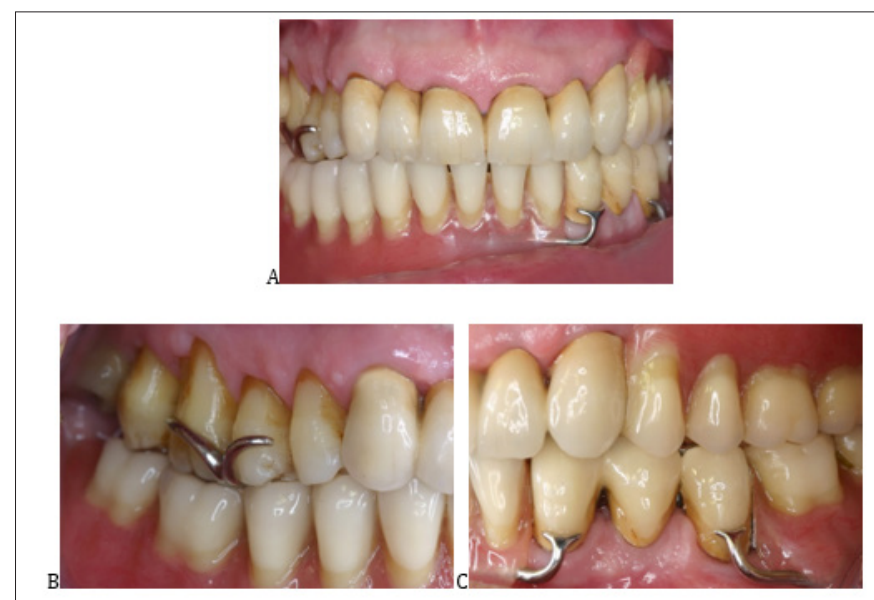

Figure 4: Definitive restorations.
A. Frontal view.
B. B, C Lateral views.

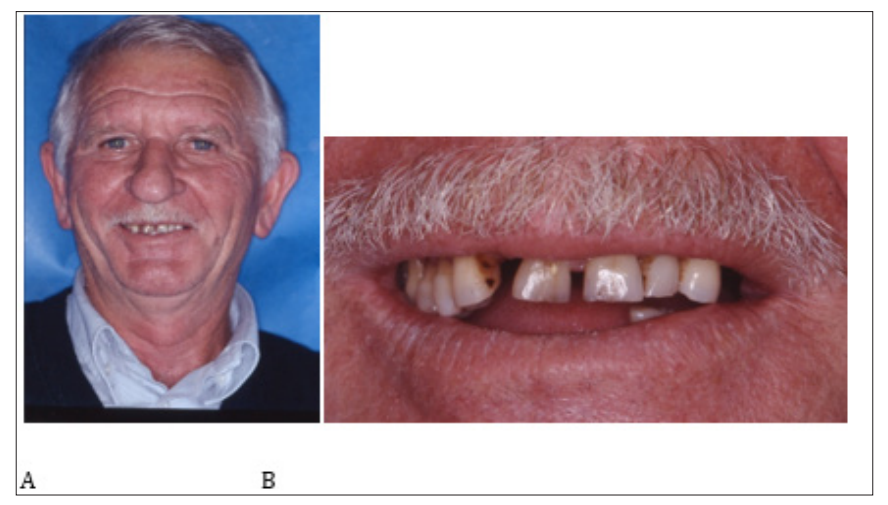

Figure 5: Extraoral preoperative condition.

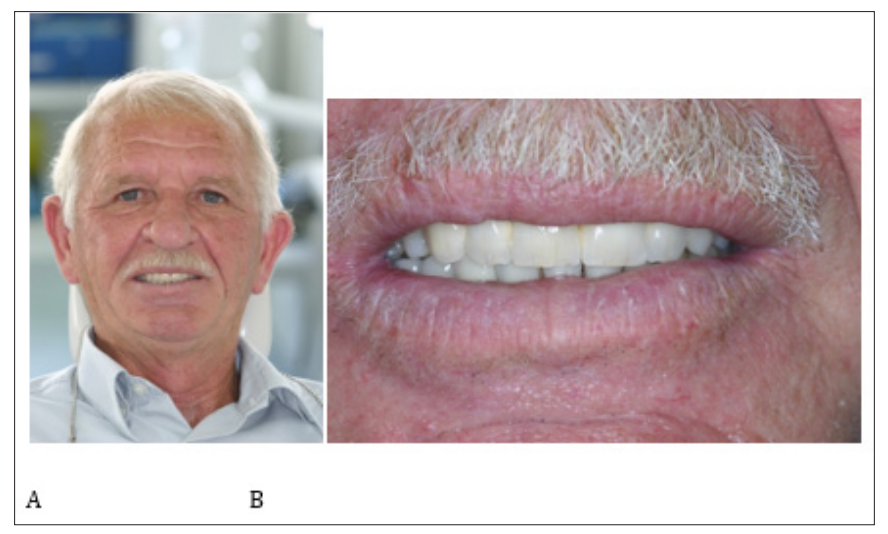

Figure 6: Extraoral post-operative condition.

\section{Discussion}

In this case report, an alternative, conservative approach for the correction of maxillary occlusal plane discrepancy was implemented, without compromising long-term prosthetic prognosis. 
Over eruption is a common problem encountered when opposing teeth are not present for extended periods of time [1]. The basic guideline for prosthodontic treatment in these complicated cases is to correct occlusal disharmony by decreasing this occlusal plane angulation [15].

The selected conservative prosthetic protocol followed in this case, offered certain clinical advantages. First, the treatment plan was less invasive and required minimum reduction of dental tissue. Fewer teeth were prepared and right maxillary teeth were left intact. As a result, no root canal treatment and no surgical procedures (crown lengthening for maxillary right teeth) were performed. Minimizing the number of metal ceramic crowns, the number of root canals and surgical procedures, significantly reduced the final cost for the patient [18]. The standard occlusal plane correction protocol would involve both surgical and endodontic procedures as well as more PFM crowns, therefore it would be more expensive.

There was a need for minimal increase in vertical dimension of occlusion(1-2mm) for enhanced aesthetics. Temporary all acrylic RPDs were used for a trial period of three months to ensure esthetics and function. Posterior support was restored and neuromuscular adaption to removable appliances was also confirmed.

Splinting remaining anterior teeth into a six-unit FDP, ensured adequate support for missing right lateral incisor, minimizing the possibility of flare up and provided better stress distribution for RDP support. An alternative design of two 3-unit separate FDPs (centrals to canines) was not advocated since it would not provide protection against anterior teeth flare-out.

Patients with multiple missing teeth regain their self-confidence when the final prosthetic restoration is esthetically acceptable [15]. The use of an ERA semi-precision attachment offered an aesthetic result without the unacceptable metal display of a conventional clasp design RPD [12,16,17]. A unilaterally positioned ERA attachment functions as a rigid connector since its vertical resiliency was restricted by the major connector (palatal zone) and the occlusal rests on maxillary molars. A Swing lock design was not proposed as alternative treatment option due to reduced available vestibular depth, the presence of extended frenum attachment and the anticipated high torque values [14].

Other possible alternative treatment plans would involve either the use of dental implants or the fabrication of an RPD with conventional metal clasps. Implant supported FDPs were rejected due to cost and conventional RDPs due to esthetics. The patient wanted to avoid a metal clasp on the canine that would be visible at smile, and the all-acrylic interim RDP did not satisfy his aesthetic demands [15].

The prosthetic complications in this case report were a clasp failure for the mandibular RDP and an acrylic tooth detachment (left central incisor) over five years of observation time. The patient followed a strict maintenance protocol every six months for five years, which included conservative periodontal treatment, light occlusal adjustments, replacement of the male ERA attachment and evaluating the need for denture base relines.

\section{References}

1. Craddock HL, Youngson CC, Manogue M, Blance A (2007) Occlusal changes following posterior tooth loss in adults. Part 1: a study of clinical parameters associated with the extent and type of supraeruption in unopposed posterior teeth. J Prosthodont 16: 485-494.

2. Zitzmann NU, Scherrer SS, Weiger R, Lang NP, Walter C (2011) Preferences of dental care providers in maintaining compromised teeth in relation to their professional status: implants instead of periodontally involved maxillary molars? Clin Oral Implants Res 22(2): 143-150.

3. D'Souza D, Dua P (2011) Rehabilitation strategies for partially edentulous prosthodontic principles and current trends. Med J Armed Forces India 67(3): 296-298.

4. Ben-Ur Z, Shifman A, Aviv I, Gorfil C (1999) Further aspects of design for distal extension removable partial dentures based on the Kennedy classification. J Oral Rehabil 26(2): 165-169.

5. Campbell SD, Cooper L, Craddock H, Hyde TP, Nattress B, et al. (2017) Removable partial dentures: The clinical need for innovation. J Prosthet Dent 118(3): 273-280.

6. Aviv I, Ben-Ur Z, Cardash HS (1989) An analysis of rotational movement of asymmetrical distal-extension removable partial dentures. J Prosthet Dent 61(2): 211-214.

7. Altay OT, Tsolka P, Preiskel HW (1990) Abutment teeth with extracoronal attachments: the effects of splinting on tooth movement. Int J Prosthodont 3(5): 441-448.

8. Burns DR, Unger JW (1994) The construction of crowns for removable partial denture abutment teeth. Quintessence Int 25(7): 471-475.

9. Charkawi HG, Wakad MT (1996) Effect of splinting on load distribution of extracoronal attachment with distal extension prosthesis in vitro. J Prosthet Dent 76(3): 315-320.

10. Itoh H, Caputo AA, Wylie R, Berg T (1998) Effects of periodontal support and fixed splinting on load transfer by removable partial dentures. J Prosthet Dent 79(4): 465-471.

11. Ma PS, Brudvik JS (2008) Managing the maxillary partially edentulous patient with extensive anterior tooth loss and advanced periodontal disease using a removable partial denture: a clinical report. J Prosthet Dent 100(4): 259-263.

12. Wang H, Zhang Y, Yao D, Chen J, et al (2011) Effects of rigid and nonrigid extracoronal attachments on supporting tissues in extension base partial removable dental prostheses: A nonlinear finite element study. J Prosthet Dent 105(5): 338-346.

13. Berg T, Caputo AA (1992) Load transfer by a maxillary distal-extension removable partial denture with cap and ring extracoronal attachments. J Prosthet Dent 68(5): 784-789.

14. Brudvik JS (1999) Advanced removable partial dentures. Quintessence, Chicago, USA pp. 107-110.

15. Beaumont AJ (2002) An overview of esthetics with removable partial dentures. Quintessence Int 33(10): 747-755.

16. Krol AJ (1973) Clasp design for extension-base removable partial dentures. J Prosthet Dent 29(4): 408-415. 
17. Donovan TE, Cho GC (2003) Esthetic considerations with removable partial dentures. J Calif Dent Assoc 31(7): 551-557.
18. Wagner B, Kern M (2000) Clinical evaluation of removable partial dentures 10 years after insertion: success rates, hygienic problems, and technical failures. Clin Oral Investig 4(2): 74-80. 